\title{
Gender differences in the long-term effects of chronic prenatal stress on the HPA axis and hypothalamic structure in rats
}

\author{
Cristina García-Cáceres ${ }^{a}$, Natalia Lagunas ${ }^{\text {b }}$, Isabel Calmarza-Font ${ }^{\text {b }}$, \\ Iñigo Azcoitia ${ }^{c}$, Yolanda Diz-Chaves ${ }^{b}$, Luis M. García-Segura ${ }^{b}$, \\ Eva Baquedano ${ }^{\text {a }}$, Laura M. Frago ${ }^{a}$, Jesús Argente ${ }^{a}$, Julie A. Chowen ${ }^{a, *}$
}

\author{
${ }^{a}$ Hospital Infantil Universitario Niño Jesús, Universidad Autónoma de Madrid, CIBER Fisiopatología de Obesidad y Nutrición \\ (CIBERobn), Instituto de Salud Carlos III, Madrid 28009, Spain \\ ${ }^{\mathrm{b}}$ Instituto Cajal, CSIC. Avenida Dr. Arce 37, Madrid 28002, Spain \\ ' Departamento de Biología Celular, Facultad de Biología, Universidad Complutense de Madrid, Madrid 28040, Spain
}

Received 22 December 2009; received in revised form 19 April 2010; accepted 20 May 2010

\section{KEYWORDS \\ Immobilization stress; \\ HPA axis; \\ Hypothalamus; \\ Cell turnover; \\ Gender; \\ Synaptic proteins; \\ Astrocytes}

\begin{abstract}
Summary Stress during pregnancy can impair biological and behavioral responses in the adult offspring and some of these effects are associated with structural changes in specific brain regions. Furthermore, these outcomes can vary according to strain, gender, and type and duration of the maternal stress. Indeed, early stress can induce sexually dimorphic long-term effects on diverse endocrine axes, including subsequent responses to stress. However, whether hypothalamic structural modifications are associated with these endocrine disruptions has not been reported. Thus, we examined the gender differences in the long-term effects of prenatal and adult immobilization stress on the hypothalamic-pituitary-adrenocortical (HPA) axis and the associated changes in hypothalamic structural proteins. Pregnant Wistar rats were subjected to immobilization stress three times daily ( $45 \mathrm{~min}$ each) during the last week of gestation. One half of the offspring were subjected to the same regimen of stress on 10 consecutive days starting at postnatal day (PND) 90. At sacrifice (PND 180), serum corticosterone levels were significantly higher in females compared to males and increased significantly in females subjected to both stresses with no change in males. Prenatal stress increased pituitary ACTH content in males, with no effect in females. Hypothalamic CRH mRNA levels were significantly increased by prenatal stress in females, but decreased in male rats. In females neither stress affected hypothalamic cell death, as determined by cytoplasmic histone-associated DNA fragment levels or proliferation, determined by proliferating cell nuclear antigen levels (PCNA); however, in males there was a significant decrease in cell death in response to prenatal stress and a decrease in PCNA levels with
\end{abstract}

* Corresponding author at: Servicio de Endocrinología, Hospital Infantil Universitario Niño Jesús, Avenida Menéndez Pelayo 65, Madrid 28009, Spain. Tel.: +34 91503 5939; fax: +34 915035939.

E-mail address: jachowen@telefonica.net (J.A. Chowen). 
both prenatal and adult stress. In all groups BrdU immunoreactivity colocalized in glial fibrillary acidic protein (GFAP) positive cells, with few BrdU/NeuN labelled cells found. Furthermore, in males the astrocyte marker $\mathrm{S} 100 \beta$ increased with prenatal stress and decreased with adult stress, suggesting affectation of astrocytes. Synapsin-1 levels were increased by adult stress in females and by prenatal stress in males, while, PSD95 levels were increased in females and decreased in males by both prenatal and adult stress. In conclusion, hypothalamic structural rearrangement appears to be involved in the long-term endocrine outcomes observed after both chronic prenatal and adult stresses. Furthermore, many of these changes are not only different between males and females, but opposite, which could underlie the gender differences in the long-term sequale of chronic stress, including subsequent responses to stress.

(C) 2010 Elsevier Ltd. All rights reserved.

\section{Introduction}

Exposure of pregnant humans or animals to chronic stress during critical periods of fetal brain development may raise the risk of depression, attention and learning deficits, hormonal imbalances and metabolic disorders in the offspring, some of which have been associated with structural alterations in the brain (Schneider, 1992; Van Os and Selten, 1998; Geddes, 1999; Rhees et al., 1999a; Weinstock, 2001; Wadhwa et al., 2001; Linnet et al., 2003; Maccari et al., 2003; Walker, 2005; Abe et al., 2007; Yaka et al., 2007; Bogoch et al., 2007). Indeed, behavioral disturbances produced by prenatal stress are associated with cellular and synaptic protein alterations in the hippocampus, dentate gyrus and prefrontal cortex of adult offspring (Lemaire et al., 2000; Koo et al. , 2003; Van den Hove et al., 2006; Michelsen et al. , 2007). Furthermore, many of the long-term behavioral alterations caused by prenatal stress, as well as the structural alterations in brain areas controlling behavior, are gender specific (Reznikov et al., 1999; Rhees et al., 1999a; Bowman et al., 2004; Tobe et al., 2005; Weinstock, 2007; Mueller and Bale, 2008; Zuena et al., 2008).

Prenatal stress also has long-term effects on distinct endocrine axes (Kofman, 2002; Mairesse et al., 2008; Mueller and Bale, 2008), but whether modifications in hypothalamic structures are involved in this process remains largely unknown. Moreover, some of these endocrine alterations in response to early stress are sexually dimorphic (Horst et al., 2009; García-Cáceres et al., 2010). As normal hypothalamic development differs between males and females, resulting in sexually dimorphic hypothalamic structures and functions (Ward, 1972; Rhees et al., 1999a,b), it follows that environmental challenges or changes during this critical period may differentially affect each gender. Hence, it is conceivable that maternal and early stresses not only induce structural changes in the hypothalamus, but that these effects are sexually dimorphic and this could contribute to the gender differences in endocrine outcomes.

As little information is available in the literature regarding the possible structural alterations in the hypothalamus in response to chronic stress or whether these changes are sexually dimorphic, our aims in this study were to: (1) analyze the effect of maternal stress on hypothalamic cell turnover and synaptic density in the adult offspring, (2) determine whether these structural changes are different between males and females, (3) examine whether exposure to a second chronic stress during adulthood results in long-lasting hormonal and hypothalamic alterations and if exposure to chronic prenatal stress modulates this response and (4) determine whether the long-term response of the hypothalamic-pituitary-adrenal axis to maternal stress, which is different between males and females, can be correlated with structural changes in the hypothalamus.

\section{Materials and methods}

\subsection{Materials}

All chemicals and reagents were purchased from Sigma Chemical Co. (St. Louis, MO) or Merck (Barcelona, Spain) unless otherwise indicated.

\subsection{Animals}

All experiments were designed according to the European Union laws for animal care and the study was approved by the local institutional Ethical Committee. Young adult pregnant Wistar rats were housed individually under alternate light $(12 \mathrm{~h})$-dark $(12 \mathrm{~h})$ periods and allowed free access to rat chow and tap water.

\subsection{Experimental design}

Prenatal restraint stress was performed daily in pregnant rats during the last week of gestation (gestational days 14-21) by placing them in transparent plastic cylinders $(7 \mathrm{~cm}$ inner diameter, $19 \mathrm{~cm}$ long) along with bright light exposition, for $45 \mathrm{~min}$, three times a day, as previously described (Ward and Weisz, 1980). Female rats from the control group remained undisturbed in their home cage.

At birth pups were housed with their mother with no handling of either the pups or the mothers until postnatal day 21 (P21) at which time they were weaned. Only litters of 9-14 pups were employed in the study. At P21, pups were distributed (four/cage) according to origin from control or stressed dams, with males and females being housed separately. At approximately P90, 9-10 animals from each of the two groups (control; C or prenatally stressed; PnS) for both sexes were subjected to adult stress (AS). Female rats were subjected to stress starting on day 2 of diestrus (as determined by daily vaginal swab). Adult stress was performed by using a similar protocol to that described for prenatal stress, but over 10 days. Either 2 or 14 days before sacrifice rats received i.p. injections of 5-bromo-2'-deoxyuridine (BrdU; Sigma-Aldrich, St. Louis, MO) for 2 consecutive days ( $50 \mathrm{mg} / \mathrm{kg}$ of body weight at a concentration of $10 \mathrm{mg} / \mathrm{ml}$ in sterile saline). 
All rats were sacrificed at 180 days of age between $1900 \mathrm{~h}$ and $2200 \mathrm{~h}$. Thus, females were at random stages of the estrous cycle. The brain and pituitary were removed and rapidly frozen in dry ice and stored at $-70{ }^{\circ} \mathrm{C}$ until processed. Adrenal glands were removed and weighed and trunk blood was collected, allowed to clot and the serum separated and stored at $-70{ }^{\circ} \mathrm{C}$ until processed.

This experimental design resulted in the following four groups ( $n=9-10 /$ group): C non-stressed (C), prenatally stressed (PnS), adult stressed (AS), and prenatally and adult stressed (PAS).

\subsection{Tissue preparation}

The hypothalami were isolated on ice by using the following boundaries: an anterior cut was made at the level of the optic chiasm, a posterior coronal section anterior to the mammilary bodies, two sagittal cuts parallel to the lateral ventricles, and a dorsal horizontal cut at the level of the anterior commissure. Each hypothalamus was then cut into two halves by following the sagittal axis. One half was processed for protein extraction and the other half was fixed in $4 \%$ paraformaldehyde in $0.1 \mathrm{M}$ phosphate buffer $(\mathrm{pH} 7.4)$ for $48 \mathrm{~h}$ at $4{ }^{\circ} \mathrm{C}$ and then rinsed with phosphate buffer (PB; $0.1 \mathrm{M}, \mathrm{pH} 7.4)$. Hypothalamic sections $(30 \mu \mathrm{m})$ were obtained using a vibratome (VT 1200S, Leica Microsystems, Wetzlar, Germany) and stored at $-20{ }^{\circ} \mathrm{C}$ in cryoprotector solution ( $30 \%$ sucrose, $30 \%$ etylene glycol in PB). The second half was stored at $-70{ }^{\circ} \mathrm{C}$ until processed for Western blotting or ELISA.

The pituitaries were weighed and transferred to a tube and stored at $-70{ }^{\circ} \mathrm{C}$ until processed for Western blotting.

\subsection{Protein extraction}

Tissue for ELISA was homogenized in lysis buffer provided by the manufacturer of the commercial kit (Roche Diagnostics, Mannheim, Germany). Tissue for Western blotting was homogenized on ice in $500 \mu \mathrm{l}$ of radioimmunoprecipitation assay lysis (RIPA) buffer with an EDTA-free protease inhibitor cocktail (Roche Diagnostics, Mannheim, Germany). After homogenization for Western blotting, samples were centrifuged at $14,000 \mathrm{rpm}$ for $20 \mathrm{~min}$ at $4{ }^{\circ} \mathrm{C}$. Supernatants were transferred to a new tube and total protein concentration was determined by the method of Bradford (Protein Assay; Bio-Rad Laboratories, Hercules, CA, USA).

\subsection{Cell death detection ELISA}

This photometric enzyme immunoassay for the quantitative determination of cytoplasmic histone-associated DNA fragments (mono- and oligo-nucleosomes) that are produced after induced cell death was carried out according to the manufacturer's instructions (Roche Diagnostics). Each sample was measured in duplicate in each assay. Background measurements were made and this value was subtracted from the mean value of each sample. This assay has a detection limit of approximately 50 dead cells/well and results were normalized to protein levels in each sample. The inter- and intra-assay coefficients of variation were $8.5 \%$ and $4.3 \%$, respectively.

\subsection{Western blotting}

Depending on the specific protein to be detected 10, 20 or $60 \mu \mathrm{g}$ of protein were resolved on a $12 \%$ SDS-polyacrylamide gel under denaturing conditions. The proteins were then electro-transferred to poly-vinylidene difluoride membranes (Bio-Rad). Membranes were blocked in TBS (20 mM) containing $5 \%$ nonfat dried milk or $5 \%$ bovine serum albumin (BSA) and $0.1 \%$ Tween 20 for $2 \mathrm{~h}$. Primary antibodies, used at a concentration of 1:1000, were as follows: anti-proliferating cell nuclear antigen (PCNA) was purchased from Signet Laboratories (Dedham, MA, USA), the antibody towards the astrocyte protein $S 100 \beta$ from Abcam (Cambridge, UK) and anti-neuron-specific beta-III tubulin (Tuj-1) from RD Systems (Minneapolis, MN, USA). The antibodies for the presynaptic protein synapsin-1 and the post-synaptic protein PSD95 were purchased from Calbiochem (Madison, WI, USA). Antibodies for the glial cell markers vimentin and glial fibrillary acidic protein (GFAP) were from Sigma-Aldrich (St. Louis, MO, USA). Anti- $\beta$-actin was from Santa Cruz Biotechnology (Santa Cruz, CA, USA) and anti-adrenocorticotropic hormone (ACTH) was from Bachem (Torrance, CA, USA). Membranes were incubated with primary antibodies overnight at $4{ }^{\circ} \mathrm{C}$ under agitation. The membranes were then washed and incubated with the corresponding secondary antibody conjugated with peroxidase (Pierce, Rockford, IL, USA) at a dilution of 1:2000. Bound peroxidase activity was visualized by chemiluminiscence (PerkinElmer Life Science, Boston, MA, USA) and quantified by densitometry by using a Kodak Gel Logic 1500 Image Analysis system and Molecular Imaging Software, version 4.0 (Rochester, NY, USA). All results were first normalized to actin levels in each lane and then to control values on each blot. All experiments were performed a minimum of two times.

\subsection{Immunohistochemistry}

Both single and double immunohistochemistry were carried out in free-floating sections under moderate shaking. After washing in phosphate buffer (PB, $0.1 \mathrm{M}, \mathrm{pH} 7.4)$, endogenous peroxidase was inhibited with a $50 \%$ methanol, $3 \% \mathrm{H}_{2} \mathrm{O}_{2}$ solution in $\mathrm{PB}$ for $15 \mathrm{~min}$. After washing in $\mathrm{PB}$, the sections were incubated in $2 \mathrm{~N} \mathrm{HCl}$ at $37{ }^{\circ} \mathrm{C}$ for 30 min and then blocked for 90 min at room temperature in PB with $1 \%$ Triton X-100 and 3\% BSA (blocking buffer). For single immunohistochemistry of BrdU sections were incubated for $48 \mathrm{~h}$ at $4{ }^{\circ} \mathrm{C}$ with anti-BrdU containing $5 \%$ normal goat serum (1:5000). The BrdU monoclonal antibody was developed by Stephen J. Kaufman under the auspices of the NICHD and maintained by The University of lowa, Department of Biological Sciences, lowa City, IA and obtained from the Developmental Studies Hybridoma Bank. Sections were then washed three times in PB with $0.3 \%$ bovine serum albumin (BSA) and $0.3 \%$ Triton $X$ 100 (PBT, $0.1 \mathrm{M} \mathrm{pH} \mathrm{7.4)} \mathrm{and} \mathrm{incubated} \mathrm{for} 90 \mathrm{~min}$ at room temperature with goat anti-mouse lgG biotin (1:1000). The sections were washed and then incubated for $45 \mathrm{~min}$ in avidin-biotin-peroxidase complex ( $A B C$ peroxidase staining kit, Pierce Rockford, IL). After three washes with PB, peroxidase was then detected by using 3,3'-diaminobenzidine tetrahydrochloride (DAB; Sigma) as a chromogen (0.05\% DAB, $0.02 \% \mathrm{H}_{2} \mathrm{O}_{2}$ in $\left.\mathrm{PB}\right)$. The sections were washed with $\mathrm{PB}$ and 
mounted on gelatin-coated slides, stained with methyl green and dehydrated through a series of graded alcohols, cleared in xylene, and covered with a coverslip in Depex.

For double immunohistochemistry of GFAP and BrdU, after the blocking, the sections were incubated for $24 \mathrm{~h}$ at $4{ }^{\circ} \mathrm{C}$ anti-GFAP (Sigma) at a dilution of 1:500 in blocking buffer containing $5 \%$ normal goat serum. Sections were then rinsed three times in buffer and incubated for $2 \mathrm{~h}$ at room temperature with biotinylated secondary anti-rabbit IgG antibody $(1: 1000)$, followed by incubation for $45 \mathrm{~min}$ in $A B C$ complex and detected by using $D A B$ as described above. The sections were washed three times at RT and treated with $2 \mathrm{~N} \mathrm{HCl}$ for 30 min at $37^{\circ} \mathrm{C}$. Afterwards, they were rinsed in $\mathrm{PB}$ and incubated with blocking buffer containing antiBrdU overnight at $4{ }^{\circ} \mathrm{C}$ as described above. After incubation, the sections were washed and incubated with a biotinylated secondary anti-mouse IgG antibody $(1: 1000)$ for $2 \mathrm{~h}$, rinsed and incubated for $45 \mathrm{~min}$ in $\mathrm{ABC}$, followed by $\mathrm{DAB}$ with $1 \%$ cobalt chloride and $1 \%$ nickel sulphate in sterile water. Finally, the sections were washed and mounted as described above.

Double-fluorescent immunohistochemistry for BrdU and NeuN was carried out in free-floating sections, after washed PB containing $0.3 \%$ bovine serum albumin (BSA) and $0.3 \%$ Triton X-100 (PBT, 0,1 M, pH 7.4). This same buffer was used in the subsequent washes. The sections were then blocked for 90 min at RT in PB with 1\% Triton X-100 and 3\% BSA and incubated for $48 \mathrm{~h}$ at $4{ }^{\circ} \mathrm{C}$ with anti-BrdU (1:750; Abcam) and NeuN (1:500, Chemicon International, Billerica, MA). Sections were then washed three times in buffer and incubated for 90 min at RT with goat anti-mouse IgG biotin $11: 1000$, Pierce Biotechnology). Afterwards sections were washed and incubated under dark conditions with streptavidin, Alexa Fluor 488 (1:2000, Molecular Probes, Leiden, The Netherlands) and Alexa Fluor 633 anti-sheep IgG (1:1000, Molecular Probes) for 90 min at RT and washed three times with buffer. Finally, sections were mounted and cover-slipped with Clear Mount (Electronic Microscopy Sciences, Hatfield, PA, USA).

Preliminary assays were performed to determine the concentration of antibodies to be used. For every experiment, sections for all groups were incubated in parallel. In each assay control slides consisted of omission of primary antibodies and verification that immunostaining was absent. Peroxidase-stained sections were examined with a light microscope (Zeiss Axioplan, Göttingen, Germany). Images were captured using a digital camera and processed using Image-Pro Plus software (version 5.0 for Windows; Media Cybernetics Inc., Silver Spring, MD). Immunofluorescence was visualized by using a confocal microscope (Leica model DMIRB; Leica, Wetzlar, Germany).

\subsection{RNA extraction and real-time PCR}

Total RNA was extracted from the hypothalami according to Tri-Reagent protocol (Chomczynski, 1993). Briefly, quantitative real-time PCR was performed on CDNA prepared from $1 \mu \mathrm{g}$ of total RNA isolated from hypothalami by reverse transcription (High capacity cDNA RT kit, Applied Biosystems, Foster City, CA, USA). Predesigned primers for corticotrophin-releasing hormone $(\mathrm{CRH})$ and $\beta$-actin and assay-ondemand kits using TaqMan Universal PCR Master Mix (Applied Biosystems) were used according to the manufacturer's pro- tocol, and analyzed by using the ABI PRISM 7000HT Sequence Detection System (Applied Biosystems). Values were normalized to the reference gene actin. According to manufacturer's guidelines, the $\Delta \Delta C_{\mathrm{T}}$ method was used for relative quantification. Statistics were performed using $\Delta \Delta C_{T}$ values.

\subsection{Determination of serum corticosterone levels by RIA}

Levels of corticosterone were measured by radioimmunoassay following the manufacturer's instructions (MP Biomedicals, Orangeburg, NY, USA). The sensitivity of the method was $7.7 \mathrm{ng} / \mathrm{ml}$ and the intra- and inter-assay variations were $7.3 \%$ and $6.9 \%$, respectively.

\subsection{Statistical analysis}

To determine if there was an interaction between sex and stress on a parameter, 2-way ANOVAs were performed. Oneway ANOVAs were performed when appropriate and post hoc comparisons were made using the Scheffe $f$-test. All data are presented as mean \pm SEM. The values were considered significantly different when the $p$ value was lower than 0.05 . Statistics were performed using the statistics program StatView (version 4.0).

\section{Results}

\subsection{Circulating corticosterone concentrations}

Serum corticosterone levels were found to be significantly affected by both sex ( $F: 108.827 ; p<0.0001)$ and stress $(F$ : 3.022; $p<0.05)$, with a significant interaction between these two factors $(F: 3.579 ; p<0.02)$. Control female rats had significantly higher corticosterone levels than control males. In males, there was no difference in corticosterone levels regardless of their previous stress experience. In contrast, corticosterone levels were significantly higher in females that had been previously exposed to the combination of prenatal and adult stress compared to control females or females that were only prenatally stressed, even though the last immobilization stress was performed 90 days before sacrifice (Fig. 1A).

\subsection{Adrenal gland weight}

There was no effect of prenatal or adult stress on adrenal gland weight in either males or females, but there was a significant effect of sex with females having significantly larger adrenal glands than males (female control: $0.232 \pm 0.010 \mathrm{~g}$, PnS females: $0.235 \pm 0.015 \mathrm{~g}$, AS females: $0.206 \pm 0.013 \mathrm{~g}$, APS females: $0.235 \pm 0.016 \mathrm{~g}$, male control: $0.118 \pm 0.010 \mathrm{~g}$, PnS males: $0.108 \pm 0.003 \mathrm{~g}$, AS males: $0.108 \pm 0.005 \mathrm{~g}$, APS males: $0.108 \pm 0.010 \mathrm{~g} ; \mathrm{F}: 233.347$; $p<0.0001, n=9-10$ /group).

\subsection{Pituitary ACTH content}

In female rats, there was no significant effect of prenatal or adult stress on pituitary ACTH content (Fig. 1B). In males, 

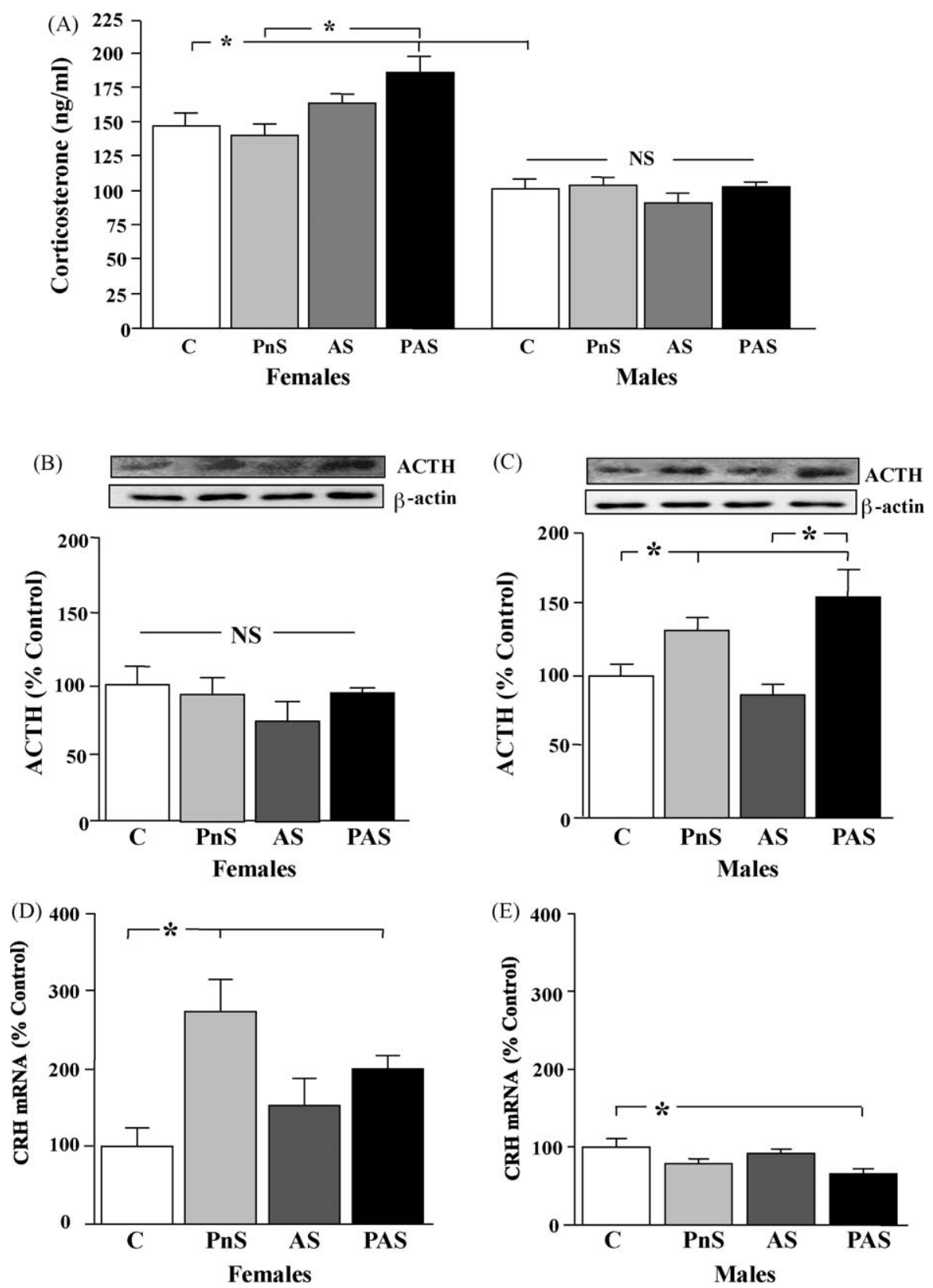

Figure 1 Circulating levels of corticosterone in female and male rats in response to stress, $n=9-10 /$ group (A). Changes in adrenocorticotropic hormone (ACTH) levels in the pituitary of female (B) and male (C) rats in response to stress ( $n=4-5 / g r o u p)$. Effect of prenatal and adult stress on hypothalamic corticotrophin-releasing hormone (CRH) mRNA levels in females (D) and males (E), $n=4-5$ /group. $C$, control; PnS, prenatal stress; AS, adult stress; PAS, prenatal and adult stress. NS, non-significant. * ANOVA $p<0.05$.

prenatal stress increased ACTH levels ( $F: 18.120 ; p<0.002)$, with no effect of adult stress (Fig. 1C).

\subsection{Hypothalamic CRH mRNA}

In female rats PnS induced a significant increase in hypothalamic CRH mRNA levels ( $F: 13.093 ; p<0.01)$ with no effect of AS (Fig. 1D). In males, there was a significant effect of prenatal stress on CRH mRNA levels $(F: 10.353, p<0.005)$ by 2-way ANOVA with no significant interaction between the two stresses. Males exposed to prenatal stress had a decline in $\mathrm{CRH}$ mRNA levels with this reaching significance when exposed to both stresses (Fig. 1E; $p<0.02$ ).

\subsection{Hypothalamic cell turnover}

In female rats, stress had no significant effect on cell death as represented by cytoplasmic histone-associated DNA fragment levels (Fig. 2A). In contrast, in male rats PnS and the combination of both stresses significantly reduced this cell death marker in the hypothalamus compared to controls (Fig. 2B; F: 5.700, $p<0.04$ ). 

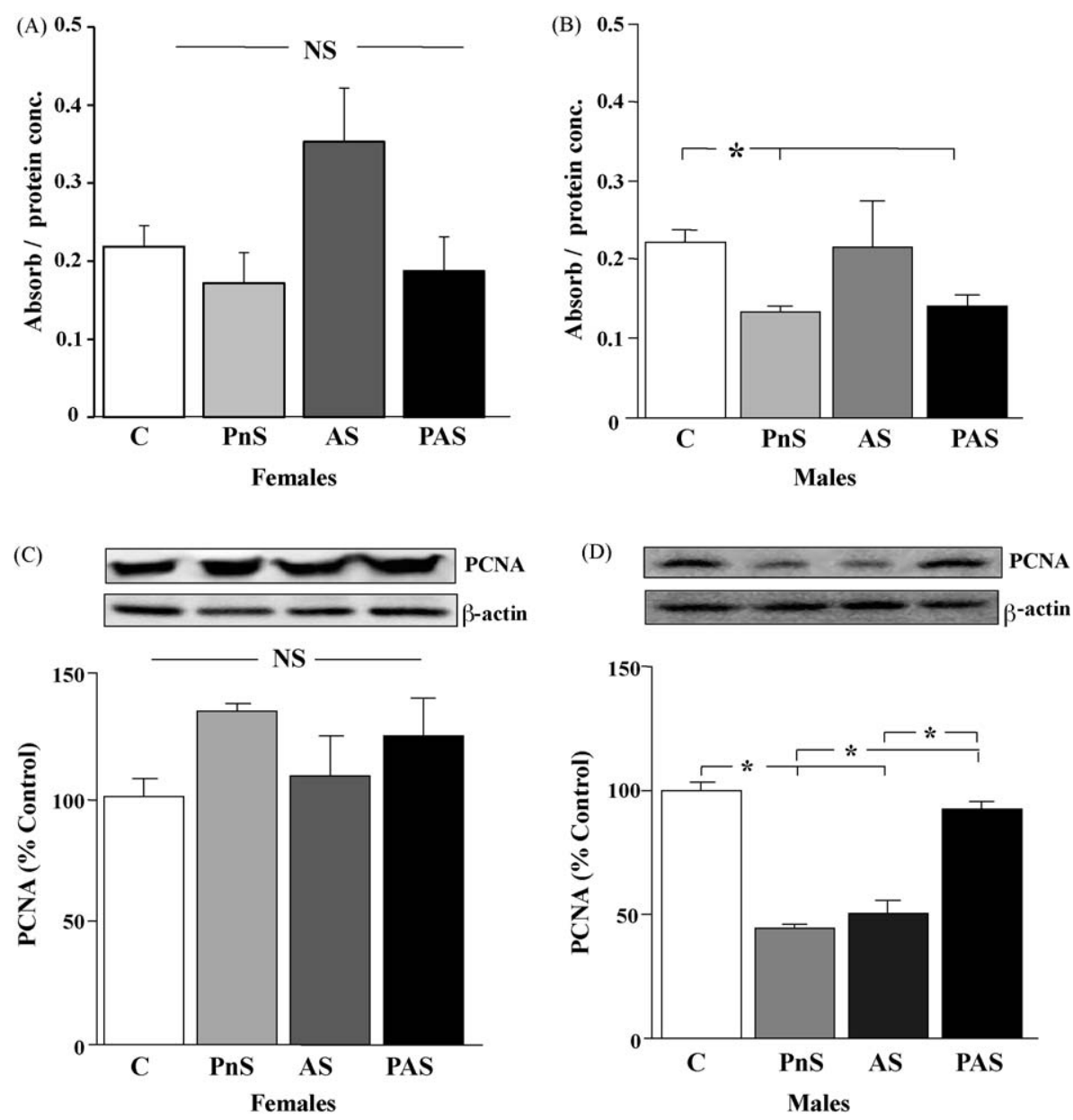

Figure 2 Effect of prenatal and adult stress on hypothalamic cell death in female (A) and male (B) rats. Changes in proliferating cell nuclear antigen (PCNA) levels in the hypothalamus of females (C) and males (D) in response to stress. C, control; PnS, prenatal stress; AS, adult stress; PAS, prenatal and adult stress. NS, non-significant. * ANOVA $p<0.05 ; n=4-5$ /group.

Stress had no effect on hypothalamic cell proliferation, as represented by PCNA levels, in the hypothalamus of female rats (Fig. $2 \mathrm{C}$ ). However, in males there was an interaction between the two stresses $(F: 9.140 ; p<0.01)$ with both prenatal and adult stress significantly reducing PCNA levels and the combination of stresses returning them to control levels (Fig. 2D).

The distribution of BrdU positive cells in the hypothalamus was similar between all experimental groups, with sparse cells being found in the lateral hypothalamus, dorsomedial hypothalamus, anterior hypothalamus and in the white matter. Slightly higher densities of BrdU positive cells were found lining and close to the 3rd ventricle and in the arcuate, supraoptic and paraventricular nuclei.

Cells immunolabeled for both BrdU and GFAP were found (Fig. 3A), with very few cells found double labelled for BrdU and NeuN in any of the experimental groups.

\subsection{Glial and neuronal markers in the hypothalamus}

We found no change in neuron-specific beta-III tubulin (Tuj-1) levels in response to either stress in males or females (data not shown). However, the effect of chronic stress on astrocyte marker levels differed according to sex. In females, there was an interaction between prenatal and adult stress on GFAP levels with the combination of stresses inducing a significant decrease (C: $100 \pm 8.8$, PnS: $128.0 \pm 12.6$, AS: $122.7 \pm 8.3$, PAS: $77.9 \pm 14.9 ; F: 10.536 ; p<0.01 ; n=4-5 /$ group). In contrast, in males GFAP levels were unchanged in response to stress (C: $100 \pm 21.5$, PnS: $111.9 \pm 26.5$, AS: $94.2 \pm 17.1$, PAS: $88.4 \pm 14.2 ; n=4-5$ /group). Hypothalamic vimentin levels were highly variable and no significant differences were found between any of the experimental groups (males C: $100 \pm 17.8, \quad \mathrm{PnS}: 95.7 \pm 23.5$, AS: $88.8 \pm 26.8$, PAS: $53.4 \pm 18.1$; females C: $100 \pm 20.8$, PnS: $172.6 \pm 92.7$, AS: $163.7 \pm 62.8$, PAS: $92.5 \pm 33.7 ; n=4-5 /$ group).

There was no effect of prenatal or adult stress on $\mathrm{S} 100 \beta$ levels in females (Fig. 3B). In contrast, both prenatal and adult stress significantly modulated $\mathrm{S} 100 \beta$ levels, a marker of mature astrocyte, in males (PnS; $F: 18.289 ; p<0.002$ and AS; F: 27.793; $p<0.001)$. Male rats exposed to prenatal stress had significantly higher and those exposed to adult stress had significantly lower $\mathrm{S} 100 \beta$ levels compared to controls. In male rats subjected to both stresses $\mathrm{S} 100 \beta$ returned to control levels (Fig. 3C; $p<0.002$ ). 

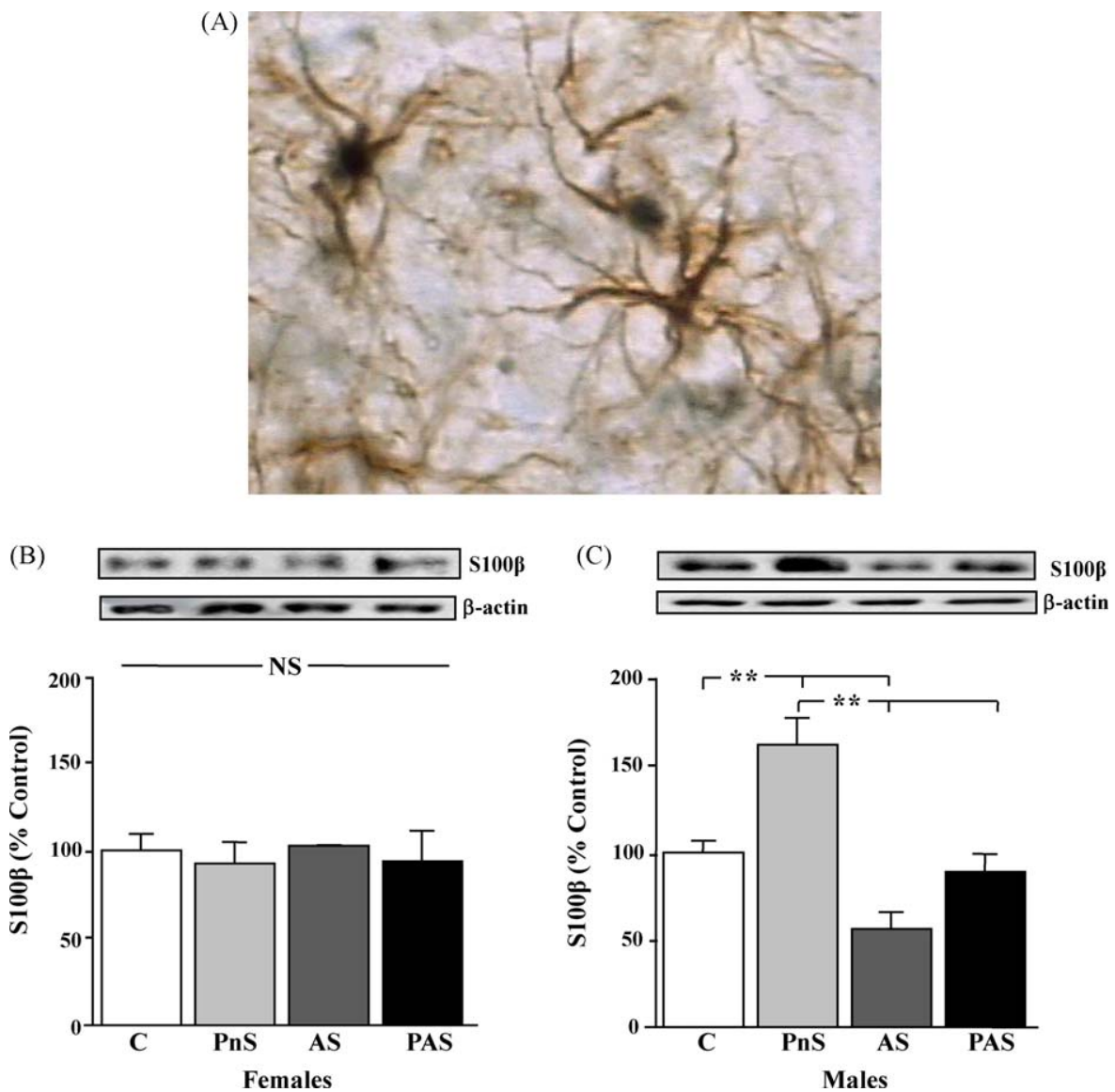

Figure 3 Immunolabeling of BrdU (black) was found to colocalize with glial fibrillary acidic protein (GFAP; brown) in cells of the hypothalamus of male rats (A). Effect of prenatal and adult stress on hypothalamic S100 $\beta$ levels of female (B) and male (C) rats. C, control; PnS, prenatal stress; AS, adult stress; PAS, prenatal and adult stress. NS, non-significant. ${ }^{* *}$, ANOVA $p<0.005$.

\subsection{Determination of synaptic proteins levels in the hypothalamus}

The effect of stress on hypothalamic synaptic proteins was also sexually dimorphic. In females, adult stress increased synapsin-1 levels $(F: 18.702 ; p<0.002)$, with prenatal stress having no effect (Fig. 4A). In contrast, in males prenatal stress increased synapsin-1 levels, with adult stress having no effect (Fig. 4B; $p<0.005$ ).

The effect of stress on the relative levels of the postsynaptic protein PSD95 was also sexually dimorphic. In female rats prenatal stress, adult stress, and the combination of these two stresses all increased PSD95 levels (Fig. 4C; $p<0.03$ ). In contrast, there was a decrease in PSD95 in male rats in response to prenatal and adult stress (Fig. 4D; $p<0.006$ ), while the combination of these stresses returned to control values.

\section{Discussion}

During development gonadal steroids play a key role in the determination of sexually dimorphic brain structures (Patchev et al., 1995, 1999; Weinstock, 2007; Zuena et al., 2008; Koehl et al., 2009) and this underlies, at least in part, the differences found between males and females in neuroendocrine functions and behavior. As brain development is different between the sexes, it follows that an insult during development may have variable outcomes. Indeed, long-term responses to different types of stress have been shown to be sexually dimorphic (Rhees et al., 1999a; Weinstock, 2007; Zuena et al., 2008; Andreano and Cahill, 2009; Bowman et al., 2009), although the mechanisms underlying this phenomenon remain largely unknown. Here we report that not only is the hypothalamic-pituitary-adrenal axis modulated differently in male and female rats, but that both prenatal and adult stress induce changes in hypothalamic cell turnover and synaptic protein concentrations, with these modifications differing dramatically between the sexes. Hence, these results suggest that structural reorganization of the hypothalamus underlies not only the affectation of endocrine axes by chronic early stress, but that it could also participate in the sexually dimorphic long-term endocrine outcomes.

The stress axis differs between males and females not only at baseline, but also in response to different stresses (Rhees et al., 1999a; Drossopoulou et al., 2004; Weinstock, 2007; Zuena et al., 2008; Andreano and Cahill, 2009; Bowman et al., 2009), which may be due at least in part to differences in gonadal steroid levels (Lund et al., 2004). Indeed, we found corticosterone levels to be higher in control females compared to control males, indicating that 
(A)
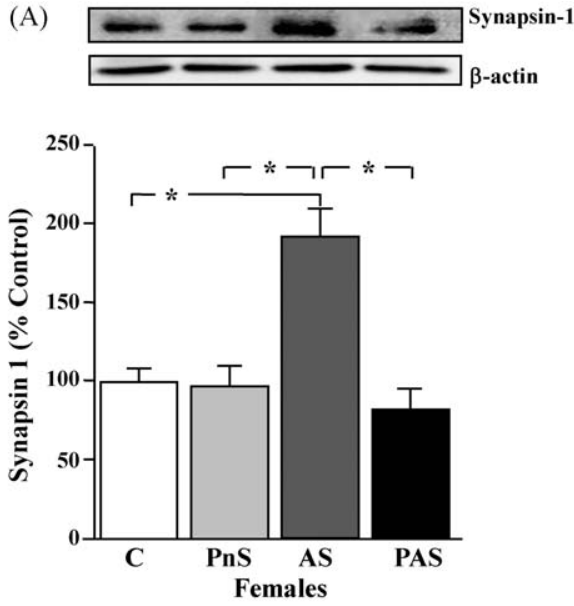

(C)
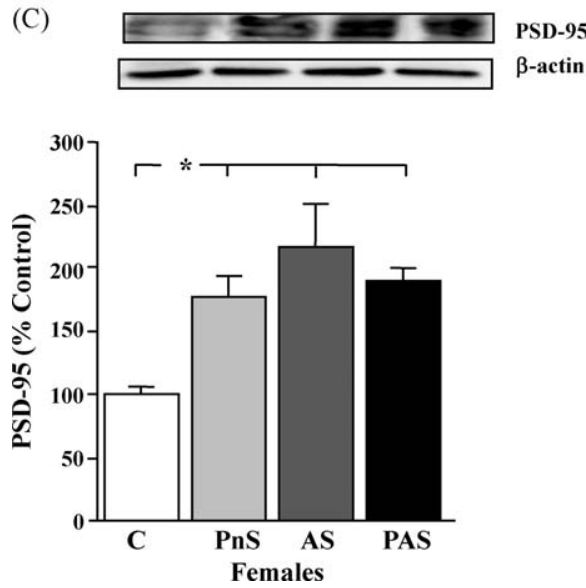

(B)
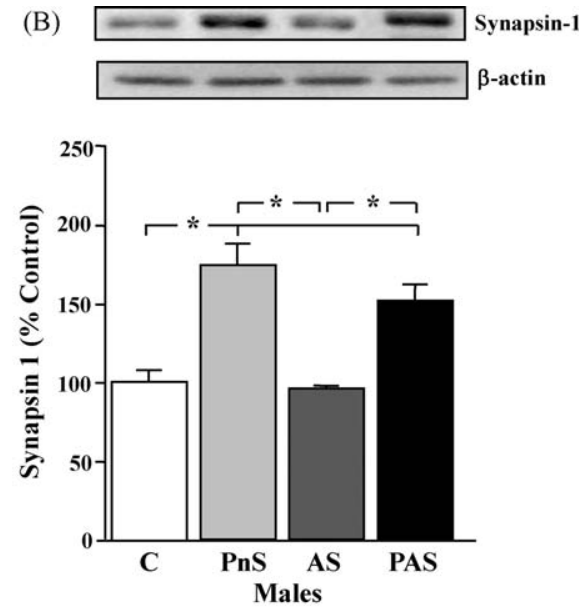

(D)
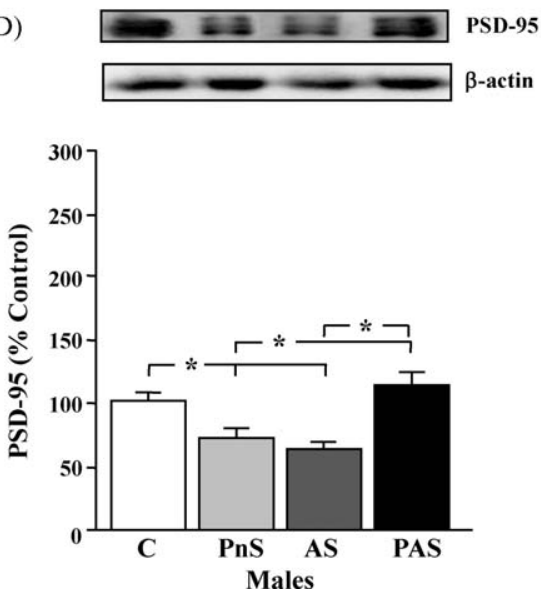

Figure 4 Effect of prenatal and adult stress on synapsin-1 and post-synaptic density protein (PSD) 95 levels in the hypothalamus of female ( $A$ and $C$, respectively) and male ( $B$ and $D$, respectively) rats. $C$, control; PnS, prenatal stress; AS, adult stress; PAS, prenatal and adult stress; NS, non-significant. * ANOVA $p<0.05 ; n=4-5 /$ group.

the basal situation of this system is different between the sexes and could participate in the differential responses to stress in the adult animal. Furthermore, in female rats corticosterone levels were increased in response to the combination of stresses, with hypothalamic CRH mRNA levels increasing in response to prenatal stress. The lack of statistical significance in response to adult stress could be due to the fact that females were sacrificed at random moments of the estrous cycle, which could underlie the high variability seen in CRH mRNA levels in female rats, as well as many of the studied variables. Increased CRH mRNA levels in response to early stress is in agreement with a previous report showing an increase in the paraventricular nucleus of adult female rats (Bosch et al., 2007) and suggests that this axis is up-regulated in females. In contrast, pituitary ACTH levels increased and hypothalamic CRH mRNA levels decreased in prenatally stressed males, suggesting a possible decrease in ACTH liberation from the anterior pituitary and a down-regulation of this axis in males. These gender specific long-term changes in the stress axis in response to early stress, as well as adult stress, could help to explain the differential responses to later stress, where females may be less susceptible to future stresses due to the up-regulation of this axis.
Modifications in hippocampal cell turnover and synaptic density have been associated with stress induced modifications in behavior (Koo et al., 2003; Bogoch et al., 2007; Darnaudéry and Maccari, 2008). Hence, we hypothesized that stress may also induce structural changes in the hypothalamus that could be associated with the endocrine outcomes. Indeed, we found that markers of cell turnover and synaptic density were affected long-term by both prenatal and adult stress. This hypothesis is further supported by the fact that the majority of changes in structural proteins were gender specific, as are many of the reported long-term endocrine responses to stress (Reznikov and Nosenko, 2000; Matthews, 2002; Luine, 2002; Welberg et al., 2006; Darnaudéry and Maccari, 2008; Mueller and Bale, 2008; García-Cáceres et al., 2010).

Prenatal stress significantly decreased both cell death and proliferation markers in the hypothalamus of male rats, suggesting a decrease in basal cell turnover. In contrast, no effect of either type of stress was observed in females. Decreased neurogenesis in the hippocampus of adult rats as a result of early life experiences has been suggested to inhibit the structural plasticity and diminish the ability of the hippocampus to respond to stress in adulthood (Mirescu et al., 2004). However, how the 
functioning of the hypothalamus and its response to further stresses is affected by this change in cell turnover is unknown, but could also indicate a modification in the ability to respond to future challenges. It is interesting to note that cell proliferation, as indicated by PCNA levels, was decreased by both prenatal and adult stresses, while the combination of these two stresses normalized this parameter. The normalization of hypothalamic cell turnover could possibly participate in the habituation of some endocrine parameters to immobilization stress (Girotti et al., 2006; García-Cáceres et al., 2010).

In the adult hypothalamus the majority of cells undergoing turnover have been reported to be glial cells (GarciaSegura and McCarthy, 2003) and we found BrdU to be incorporated into GFAP positive astrocytes. Gliosis, as indicated by increased expression of GFAP and vimentin, in the cortex and hippocampus after chronic immobilization stress (Barros et al., 2006; Jang et al., 2008) is suggested to indicate neurodegeneration (Jang et al., 2008). However, in the hypothalamus there was no change in vimentin levels and GFAP levels actually decreased in females in response to both prenatal and adult stress, with no effect in males. Thus, gliosis does not appear to occur in the hypothalamus in response to immobilization stress. Previous studies have demonstrated that $\mathrm{S} 100 \beta$, an astrocyte-derived protein, can also be increased in response to neurodegeneration, inflammation and in psychiatric diseases (Rothermundt et al., 2003; Mrak and Griffin, 2005; Craft et al., 2005; Ralay et al., 2006) and prenatal stress resulted in increased hypothalamic $S 100 \beta$ levels in males. Therefore, increased $\mathrm{S} 100 \beta$ levels in response to maternal stress could represent increased susceptibility to pathologic processes in males. However, adult stress decreased S100 $\beta$ levels and the combination of the two stresses returned them to control levels. The inverse changes in hypothalamic $\mathrm{S} 100 \beta$ in response to prenatal and adult stress could be due to the age of the animals at the moment of stress, as $\mathrm{S} 100 \beta$ has also been reported to be a regulator of neurite outgrowth and to enhance survival of neurons only during development (Rothermundt et al., 2003). Thus, the changes in this glial marker could be related to other astrocytic functions as astrocytes are involved in many aspects of neuronal function (Pellerin et al., 2007; Brown and Ransom, 2007; Milligan and Watkins, 2009) and also participate in the regulation of synaptic changes in the hypothalamus (Theodosis et al., 2006; Prevot et al., 2007; Garcia-Segura et al., 2008; Ojeda et al., 2008). Hence, changes in glial cells could be associated with the changes in synaptic protein levels.

Synapsin-1, a presynaptic protein related to the number of synaptic inputs, was significantly increased by prenatal stress in males and by adult stress in females. Inverse effects in males and females were also seen with PSD95, with both prenatal and adult stress increasing this protein in females and decreasing it in males. It is clear that synaptic protein density is affected and that this occurs in a sexually dimorphic manner even though the hypothalamic neuronal systems where these synaptic changes are occurring cannot be determined as these parameters were analyzed in whole hypothalamic homogenates. However, the modifications in PSD95 levels indi- cate that at least some of the synapses involved are glutamatergic and that these stimulatory inputs may be increased in females and decreased in males in response to both prenatal and adult stress. Indeed, prenatal stress alters synaptic inputs in the frontal cortex, striatum and hippocampus (Barros et al., 2006; Michelsen et al., 2007), with the responsiveness of the glutamatergic system in the prefrontal cortex known to be affected (Fumagalli et al., 2009). Whether these changes in hypothalamic excitatory inputs are related to the increase in the activation of the stress axis, including the increase in CRH mRNA levels in females and the decrease in males deserves further investigation.

Early brain development is vulnerable to high levels of circulating corticosterone, which in rats has been shown to cross the placental barrier especially during the last week of gestation leading to disturbances in the formation of neural circuits (Weinstock, 2007; Mabandla et al., 2008). Indeed, chronic corticosterone exposure modulates cell morphology and cell death in various brain areas, including the hypothalamus (Skynner et al., 2006). Thus, the changes in hypothalamic synaptic proteins and cell turnover markers could be due to high levels of maternal glucocorticoids in response to prenatal stress (Barros et al., 2006) and this could underlie the neuroendocrine alterations that persist into adulthood. Early events have previously been show to induce long-term alterations in central $\mathrm{CRH}$ and corticosterone levels that affect the responsiveness of the HPA axis in the adult animal (Weaver et al., 2004; McGill et al., 2006; Mabandla et al., 2008; Mueller and Bale, 2008). Here we show that both prenatal and adult immobilization stress induce long-term changes in the HPA axis, as well as modifications in synaptic and structural protein levels in the hypothalamus. Furthermore, these changes are gender specific, which could help to explain some of the differences between the sexes in the physiological manifestations in response to stress and repeated stresses, as well as the pathological responses to chronic stress.

\section{Role of the funding sources}

Funding for this study was provided by grants from Fondo de Investigación Sanitaria (PI070182), Ministerio de Ciencia e Innovación (BFU2008-02950 C03-1, C03-2 and C03-3), CIBER de Fisiopatología de Obesidad y Nutrición (CIBEROBN) Instituto de Salud Carlos II and Fundación de Endocrinología y Nutrición. CG-C is supported by a predoctoral fellowship from the Ministerio de Educación y Ciencía (FPU AP2006/02761) and JAC is supported by the biomedical investigation program of the Consejería de Sanidad y Consumo de la Comunidad de Madrid.

None of the funding institution had any further role in the study design, the collection, analysis and interpretation of data, writing of the report or in the decision to submit the paper for publication. None of the authors have anything to declare.

\section{Conflict of interest}

None declared. 


\section{Acknowledgements}

The authors would like to thank Dr. Vicente Barrios for advice in performing the hormone assays and Francisca Díaz and Sandra Canelles for the excellent technical support.

\section{References}

Abe, H., Hidaka, N., Kawagoe, C., Odagiri, K., Watanabe, Y., Ikeda, T., Ishizuka, Y., Hashiguchi, H., Takeda, R., Nishimori, T., Ishida, Y., 2007. Prenatal psychological stress causes higher emotionality, depression-like behavior, and elevated activity in the hypothalamo-pituitary-adrenal axis. Neurosci. Res. 59, 145-151.

Andreano, J.M., Cahill, L., 2009. Sex influences on the neurobiology of learning and memory. Learn. Mem. 16, 248-266.

Barros, V.G., Duhalde-Vega, M., Caltana, L., Brusco, A., Antonelli, M.C., 2006. Astrocyte-neuron vulnerability to prenatal stress in the adult rat brain. J. Neurosci. Res. 83, 787-800.

Bogoch, Y., Biala, Y.N., Limial, M., Weinstock, M., 2007. Anxiety induced by prenatal stress is associated with suppression of hippocampal genes involved in synaptic function. J. Neurochem. 101, 1018-1030.

Bosch, O.J., Müsch, W., Bredewold, R., Slattery, D.A., Neumann, I.D., 2007. Prenatal stress increases HPA axis activity and impairs maternal care in lactating female offspring: implications for postpartum mood disorder. Psychoneuroendocrinology 32, 267-278.

Bowman, R.E., MacLusky, N.J., Sarmiento, Y., Frankfurt, M., Gordon, M., Luine, V.N., 2004. Sexually dimorphic effects of prenatal stress on cognition, hormonal responses, and central neurotransmitters. Endocrinology 145, 3778-3787.

Bowman, R.E., Micik, R., Gautreaux, C., Fernandez, L., Luine, V.N., 2009. Sex-dependent changes in anxiety, memory, and monoamines following one week of stress. Physiol. Behav. 97, 21-29.

Brown, A.M., Ransom, B.R., 2007. Astrocyte glycogen and brain energy metabolism. Glia 55, 1263-1271.

Chomczynski, P., 1993. A reagent for the single-step simultaneous isolation of RNA, DNA and proteins from cell and tissue samples. Biotechniques 15 (532-534), 536-537.

Craft, J.M., Watterson, D.M., Marks, A., Van Eldik, L.J., 2005. Enhanced susceptibility of S-100B transgenic mice to neuroinflammation and neuronal dysfunction induced by intracerebroventricular infusion of human beta-amyloid. Glia 51, 209-216.

Darnaudéry, M., Maccari, S., 2008. Epigenetic programming of the stress response in male and female rats by prenatal restraint stress. Brain Res. Rev. 57, 571-585.

Drossopoulou, G., Antoniou, K., Kitraki, E., Papathanasiou, G., Papalexi, E., Dalla, C., Papadopoulou-Daifoti, Z., 2004. Sex differences in behavioral, neurochemical and neuroendocrine effects induced by the forced swim test in rats. Neuroscience 126, 849-857.

Fumagalli, F., Pasini, M., Frasca, A., Drago, F., Racagni, G., Riva, M.A., 2009. Prenatal stress alters glutamatergic system responsiveness in adult rat prefrontal cortex. J. Neurochem. 109, 17331744.

García-Cáceres, C., Diz-Chaves, Y., Lagunas, N., Calmarza-Font, I.A., Azcoitia, I., Garcia-Segura, L.M., Frago, L.M., Argente, J., Chowen, J.A., 2010. The weight gain response to postnatal stress is conditioned by both sex and prenatal stress exposure. Psychoneuroendocrinology 35, 403-413.

Garcia-Segura, L.M., Lorenz, B., DonCarlos, L.L., 2008. The role of glia in the hypothalamus: implications for gonadal steroid feedback and reproductive neuroendocrine output. Reproduction 135 , 419-429.

Garcia-Segura, L.M., McCarthy, M.M., 2003. Minireview: role of glia in neuroendocrine function. Endocrinology 145, 1082-1086.
Geddes, J., 1999. Prenatal and perinatal risk factors for early onset schizophrenia affective psychosis, and reactive psychosis. BMJ 318, 426.

Girotti, M., Pace, T.W., Gaylord, R.I., Rubin, B.A., Herman, J.P., Spencer, R.L., 2006. Habituation to repeated restraint stress is associated with lack of stress-induced c-fos expression in primary sensory processing areas of the rat brain. Neuroscience 138, 1067-1081.

Horst, G.J.T., Wichmann, R.M., Gerrits, M., Westenbroek, C., Lin, Y., 2009. Sex differences in stress responses: focus on ovarian hormones. Physiol. Behav. 97, 239-249.

Jang, S., Suh, S.H., Yoo, H.S., Lee, Y.M., Oh, S., 2008. Changes in iNOS, GFAP, NR1 expression in various brain regionas and elevation of sphingosine-1-phosphate in serum after immobilizaed stress. Neurochem. Res. 33, 842-851.

Koehl, M., Lemaire, V., Le Moal, M., Abrous, D.N., 2009. Age-dependent effect of prenatal stress on hippocampal cell proliferation in female rats. Eur. J. Neurosci. 29, 635-640.

Kofman, 0., 2002. The role of prenatal stress in the etiology of developmental behavioural disorders. Neurosci. Biobehav. Rev. 26, 457-470.

Koo, J.W., Park, C.H., Choi, S.H., Kim, N.J., Kim, H.S., Choe, J.C., Suh, Y.H., 2003. The postnatal environment can counteract prenatal effects on cognitive ability, cell proliferation, and synaptic protein expression. FASEB J. 17, 1556-1558.

Lemaire, V., Koehl, M., Le Moal, M., Abrous, D.N., 2000. Prenatal stress produces learning deficits associated with an inhibition of neurogenesis in the hippocampus. Proc. Natl. Acad. Sci. U.S.A. 97, 11032-11037.

Linnet, K.M., Dalsgaard, S., Obel, C., Wisborg, K., Henriksen, T.B., Rodriguez, A., Kotimaa, A., Moilanen, I., Thomsen, P.H., Olsen, J., Jarvelin, M.R., 2003. Maternal lifestyle factors in pregnancy risk of attention deficit hyperactivity disorder and associated behaviors: review of the current evidence. Am. J. Psychiatry 160, 1028-1040.

Luine, V., 2002. Sex differences in chronic stress effects on memory in rats. Stress $5,205-216$

Lund, T.D., Munson, D.J., Haldy, M.E., Handa, R.J., 2004. Androgen inhibits, while oestrogen enhances, restraint-induced activation of neuropeptide neurones in the paraventricular nucleus of the hypothalamus. J. Neuroendocrinol. 16, 272-278.

Mabandla, M.V., Dobson, B., Johnson, S., Kellaway, L.A., Daniels, W.M., Russell, V.A., 2008. Development of a mild prenatal stress rat model to study long term effects on neural function and survival. Metab. Brain Dis. 23, 31-42.

Maccari, S., Darnaudery, M., Morley-Fletcher, S., Zuena, A.R., Cinque, C., Van-Reeth, O., 2003. Prenatal stress and long-term consequences: implications of glucocorticoid hormones. Neurosci. Biobehav. Rev. 27, 119-127.

Mairesse, J., Viltart, O., Salomé, N., Giuliani, A., Catalani, A., Casolini, P., Morley-Fletcher, S., Nicoletti, F., Maccari, S., 2008. Prenatal stress alters the negative correlation between neuronal activation in limbic regions and behavioral responses in rats exposed to high and low anxiogenic environments. Psychoneuroendocrinology 32, 765-776.

Matthews, S.G., 2002. Early programming of the hypothalamopituitary-adrenal axis. Trends Endocrinol. Metab. 13, 373380.

McGill, B.E., Bundle, S.F., Yaylaoglu, M.B., Carson, J.P., Thaller, C., Zoghbi, H.Y., 2006. Enhanced anxiety and stress-induced corticosterone release are associated with increased CRH expression in a mouse model of Rett syndrome. Proc. Natl. Acad. Sci. U.S.A. 103, 18267-18272.

Michelsen, K.A., Van den Hove, D.L., Schmitz, C., Segers, O., Prickaerts, J., Steinbusch, H.W., 2007. Prenatal stress and subsequent exposure to chronic mild stress influence dendritic spine density and morphology in the rat medial prefrontal cortex. BMC Neurosci. 8, 107. 
Milligan, E.D., Watkins, L.R., 2009. Pathological and protective roles of glia in chronic pain. Nat. Rev. Neurosci. 10, 23-36.

Mirescu, C., Peters, J.D., Gould, E., 2004. Early life experience alters response of adult neurogenesis to stress. Nat. Neurosci. 7 , 841-846.

Mrak, R.E., Griffin, W.S., 2005. Glia and their cytokines in progression of neurodegeneration. Neurobiol. Aging 26 (3), 349-354.

Mueller, B.R., Bale, T.L., 2008. Sex-specific programming of offspring emotionality after stress early in pregnancy. J. Neurosci. 28, 9055-9065.

Ojeda, S.R., Lomniczi, A., Sandau, U.S., 2008. Glial-gonadotrophin hormone $(\mathrm{GnRH})$ neurone interactions in the median eminence and the control of GnRH secretion. J. Neuroendocrinol. 20, 732-742.

Patchev, V.K., Hayashi, S., Orikasa, C., Almeida, O.F., 1995. Implications of estrogen-dependent brain organization for gender differences in hypothalamo-pituitary-adrenal regulation. FASEB J. 9, 419-423.

Patchev, V.K., Hayashi, S., Orikasa, C., Almeida, O.F., 1999. Ontogeny of gender-specific responsiveness to stress and glucocorticoids in the rat and its determination by the neonatal gonadal steroid environment. Stress 3, 41-54.

Pellerin, L., Bouzier-Sore, A.K., Aubert, A., Serres, S., Merle, M., Costalat, R., Magistretti, P.J., 2007. Activity-dependent regulation of energy metabolism by astrocytes: an update. Glia 55, $1251-1262$.

Prevot, V., Dehouck, B., Poulain, P., Beauvillain, J.C., Buée-Scherrer, V., Bouret, S., 2007. Neuronal-glial-endothelial interactions and cell plasticity in the postnatal hypothalamus: implications for the neuroendocrine control of reproduction. Psychoneuroendocrinology 32 (Suppl. 1), S46-51.

Ralay, R.H., Craft, J.M., Hu, W., Guo, L., Wing, L.K., Van Eldik, L.J., Watterson, D.M., 2006. Glia as a therapeutic target: selective suppression of human amyloid-beta-induced upregulation of brain proinflammatory cytokine production attenuates neurodegeneration. J. Neurosci. 26, 662-670.

Reznikov, A.G., Nosenko, N.D., Tarasenko, L.V., 1999. Prenatal stress and glucocorticoid effects on the developing gender-related brain. J. Steroid Biochem. Mol. Biol. 69, 109-115.

Reznikov, O.H., Nosenko, N.D., 2000. The perinatal stress modification of the reactivity of the hypothalamo-hypophyseal-adrenal system. Fiziol. Zh. 46, 146-158.

Rhees, B.W., Al-Saleh, H.N., Kinghorn, E.W., Fleming, D.E., Lephart, E.D., 1999a. Relationship between sexual behavior and sexually dimorphic structures in the anterior hypothalamus in control and prenatally stressed male rats. Brain Res. Bull. 50, 193-199.

Rhees, B.K., Ernst, C.A., Miao, C.H., Atchley, W.R., 1999b. Uterine and postnatal maternal effects in mice selected for differential rate of early development. Genetics 153, 905-917.

Rothermundt, M., Peters, M., Prehn, J.H., Arolt, V., 2003. S100B in brain damage and neurodegeneration. Microsc. Res. Tech. 60, 614-632.

Schneider, M.L., 1992. Prenatal stress exposure alters postnatal behavioral expression under conditions of novelty challenge in rhesus monkey infants. Dev. Psychobiol. 25, 529-540.
Skynner, H.A., Amos, D.P., Murray, F., Salim, K., Knowles, M.R. Munoz-Sanjuan, I., Camargo, L.M., Bonnert, T.P., Guest, P.C., 2006. Proteomic analysis identifies alterations in cellular morphology and cell death pathways in mouse brain after chronic corticosterone treatment. Brain Res. 1102, 12-26.

Theodosis, D.T., Trailin, A., Poulain, D.A., 2006. Remodeling of astrocytes, a prerequisite for synapse turnover in the adult brain? Insights from the oxytocin system of the hypothalamus. Am. J. Physiol. Regul. Integr. Comp. Physiol. 290, R1175-R1182.

Tobe, I., Ishida, Y., Tanaka, M., Endoh, H., Fujioka, T., Nakamura, S., 2005. Effects of repeated maternal stress on FOS expression in the hypothalamic paraventricular nucleus of fetal rats. Neuroscience 134, 387-395.

Van den Hove, D.L., Steinbusch, H.W., Scheepens, A., Van de Berg, W.D., Kooiman, L.A., Boosten, B.J., Prickaerts, J., Blanco, C.E., 2006. Prenatal stress and neonatal rat brain development. Neuroscience 137, 145-155.

Van Os, J., Selten, J.P., 1998. Prenatal exposure to maternal stress and subsequent schizophrenia. The May 1940 invasion of the Netherlands. Br. J. Psychiatry 172, 324-326.

Wadhwa, P.D., Sandman, C.A., Garite, T.J., 2001. The neurobiology of stress in human pregnancy: implications for prematurity and development of the fetal central nervous system. Prog. Brain Res. $133,131-142$

Walker, C.D., 2005. Nutritional aspects modulating brain development and the responses to stress in early neonatal life. Prog. Neuropsychopharmacol. Biol. Psychiatry 29, 1249-1263.

Ward, I.L., 1972. Prenatal stress feminizes and desmasculinizes the behavior of males. Science 175, 82-84.

Ward, I.L., Weisz, J., 1980. Maternal stress alters plasma testosterone in fetal males. Science 207, 328-329.

Weaver, I.C., Cervoni, N., Champagne, F.A., D’Alessio, A.C., Sharma, S., Seckl, J.R., Dymov, S., Szyf, M., Meaney, M.J., 2004. Epigenetic programming by maternal behavior. Nat. Neurosci. 7, 847-854.

Weinstock, M., 2001. Alterations induced by gestational stress in brain morphology and behavior of the offspring. Prog. Neurobiol. 65, 427-452.

Weinstock, M., 2007. Gender differences in the effects of prenatal stress on brain development and behaviour. Neurochem. Res. 32, 1730-1740.

Welberg, L., Thrivikraman, K.V., Plotsky, P.M., 2006. Combined preand postnatal environmental enrichment programs the HPA axis differentially in male and female rats. Psychoneuroendocrinology $31,553-564$.

Yaka, R., Salomon, S., Matzner, H., Weinstock, M., 2007. Effect of varied gestational stress on acquisition of spatial memory, hippocampal LTP and synaptic proteins in juvenile male rats. Behav. Brain Res. 179, 126-132.

Zuena, A.R., Mairesse, J., Casolini, P., Cinque, C., Alemà, G.S., Morley-Fletcher, S., Chiodi, V., Spagnoli, L.G., Gradini, R., Catalani, A., Nicoletti, F., Maccari, S., 2008. Prenatal restraint stress generates two distinct behavioral and neurochemical profiles in male and female rats. PLoS One 3, e2170. 